\title{
Reply to the letter regarding NADPH oxidase inhibitor
}

\author{
Hiroki lida $^{1} \cdot$ Kazuhiro Kito $^{1} \cdot$ Kumiko Tanabe $^{1}$ \\ Received: 28 August 2018 / Accepted: 8 September 2018 / Published online: 11 November 2018 \\ (c) Japanese Society of Anesthesiologists 2018
}

To the Editor:

The question raised here is very important on a scientific basis of our study [1]. As they pointed out, both apocynin and diphenylene iodonium (DPI) may not be selective NADPH oxidase inhibitor, especially in the vascular system [2]. In our manuscript, an important point is to show in vivo experiment that acute hyperglycemia impairs endothelium-dependent dilation in cerebral vessels through oxidative stress, not limited to NADPH oxidase. Therefore, we use both apocynin and DPI to prove the contribution of oxidative stress. Even in recent several vascular studies, apocynin or DPI is used as an NADPH inhibitor, but its use may be strictly a matter to avoid $[3,4]$. We just want to describe that controlling oxidative stress maintains the vascular response and anti-oxidative stress drugs might be useful for preventing a dysfunction of the cerebral arterioles in the endothelium caused by hyperglycemia. Therefore, if we want to verify the contribution of NADPH oxidase itself in the present study, we need to perform the additional study using selective NADPH oxidase inhibitor as they suggested.

\section{References}

1. Kito K, Tanabe K, Sakata K, Fukuoka N, Nagase K, Iida M, Iida $\mathrm{H}$. Endothelium-dependent vasodilation in the cerebral arterioles of rats deteriorates during acute hyperglycemia and then is restored by reducing the glucose level. J Anesth. 2018;32:531-8.

2. Wind S, Beuerlein K, Eucker T, Muller H, Scheurer P, Armitage ME, Ho H, Schmidt HH, Wingler K. Comparative pharmacology of chemically distinct NADPH oxidase inhibitors. Br J Pharmacol. 2010;161:885-98.

3. Clemmer JS, Xiang L, Lu S, Mittwede PN, Hester RL. Hyperglycemia-mediated oxidative stress increases pulmonary vascular permeability. Microcirculation. 2016;23:221-9.

4. El-Bassossy HM, Neamatallah T, Balamash KS, Abushareb AT, Watson ML. Arginase overexpression and NADPH oxidase stimulation underlie impaired vasodilation induced by advanced glycation end products. Biochem Biophys Res Commun. 2018;499:992-7.

This comment refers to the article available online at https://doi. org/10.1007/s00540-018-2552-2.

Hiroki Iida

iida@gifu-u.ac.jp

1 Department of Anesthesiology and Pain Medicine, Gifu University Graduate School of Medicine, Yanagido 1-1, 501-1194 Gifu, Gifu, Japan 